\title{
Light emission enhancement using randomly distributed plasmonic nanoparticle arrays
}

\author{
J. Butkus ${ }^{\mathrm{a}, *}$, A. P. Edwards ${ }^{\mathrm{a}}$, F. P. Quacquarellib and A. M. Adawi ${ }^{\mathrm{a}}{ }^{\text {, }}$ \\ ${ }^{a)}$ Department of Physics and Mathematics, University of Hull, Hull, HU6 7RX, United \\ Kingdom \\ ${ }^{b)}$ Department of Physics and Astronomy, University of Sheffield, Sheffield, S3 7RH, United \\ Kingdom
}

*Corresponding Authors: $\underline{\text { a.adawi@ hull.ac.uk and butkus11@gmail.com }}$

Keywords: Plasmonic array; metallic nano-particles; fluorescent dye

\begin{abstract}
We have fabricated and characterised the optical properties of solution processed randomly distributed gold nanoparticle plasmonic arrays that are coated with a thin-film fluorescent dye. Three times enhancement in the emission intensity of the fluorescent dye Pyridine 2 has been observed. Our results are further supported by finite difference time domain simulations that predicted up to 7 times enhancements in the emission intensity as a result of the coupling between the molecular dipoles and the confined field in the underlying plasmonic array. Our results demonstrate the potential of using such structures in organic light emitting devices and chemical and bio-sensing applications.
\end{abstract}

\section{Introduction}

(c) 2014, Elsevier. Licensed under the Creative Commons Attribution-NonCommercial-NoDerivatives 4.0 International http://creativecommons.org/licenses/by-nc-nd/4.0/ 
Localized surface plasmon resonances are collective non-propagating oscillations of conduction band electrons in metallic nanoparticles coupled into electromagnetic waves [1,2], that provide metallic nanoparticles with the ability to concentrate electromagnetic fields in sub wavelength volumes at the order of $(\lambda / 15)^{3}[3]$. These resonance frequencies can be adjusted through engineering the size and shape of the metallic nanoparticle [2,4]. There is significant interest in coupling semiconductor materials to such metallic nanoparticles, as they can be considered as an ideal system to study the fundamental physics of light matter interaction under extreme electromagnetic field confinement. Possible applications include quantum optics [5-7], light management [9-12] and chemical/biological sensing devices [8]. In particular the localized surface plasmon resonances for gold and silver have been extensively studied in systems designed for light absorption enhancement $[9,10]$ with high interest for their applications in areas such as solar power harvesting [11] and enhancement of spontaneous emission rate [12].

Periodic plasmonic nanoparticle arrays are currently being extensively studied and used in designed plasmonic systems for the use in semiconductor photovoltaic and photonic systems due to their high potential in improving device efficiency in terms of absorption [11] and spontaneous emission enhancement [13-17]. Depending on the geometrical parameters of the plasmonic nanoparticle array and the optical properties of the emitting material in use, enhancements in the spontaneous emission intensity range between 3.6 to 83 have been already achieved [13,14]. Although periodic plasmonic system effectiveness was proven, the production of such structures is a relatively slow and costly process with a real difficulty in applying nano-scale lithographic patterning methods into real large-area devices. Thermally evaporated particulate metallic films are also extensively used to enhance the emission intensity of semiconductor materials with enhancements in the range 2-22 times already achieved [18-23]. However, vacuum thermal evaporation technique is costly, poorly scalable process and not always easy to incorporate particulate metallic films into the device layers [24-26]. On the contrary to the above two approaches, solution processed randomly distributed plasmonic nanoparticle arrays manufacturing is more efficient in terms of price and production time with the ability to be applied into real large-area devices using techniques such as roll-to-roll or inkjet printing. Despite these facts they are much less studied compared to the periodical arrays or thermally evaporated particulate metallic films. Just recently Xiao et al [27] have shown that surface plasmon (C) 2014, Elsevier. Licensed under the Creative Commons Attribution-NonCommercial-NoDerivatives 4.0 International http://creativecommons.org/licenses/by-nc-nd/4.0/ 
induced enhancement of electroluminescence by around $\sim 25 \%$ in organic light emitting diode (OLED) with gold nanoparticle array embedded into the hole injection layer compared to $22 \%-28 \%$ in OLED implementing 1D and 2D periodic plasmonic arrays [28] respectively.

In this paper we present the fabrication of solution processed randomly distributed plasmonic nanoparticle arrays and demonstrate over three times enhancement in the spontaneous emission intensity of the fluorescent molecular dye Pyridine 2 by coupling the molecular dipoles into the confined optical field of the plasmonic nanoparticle array. Using three dimensional finite difference time domain (FDTD) calculations [29] we show that the emission intensity enhancements are strongly dependent on the gold fill factor (FF) with emission enhancement as high as 7 times being anticipated in structures have a FF of 0.4 .

\section{Experimental Method}

The structure we have fabricated and tested in this work is shown schematically in Fig. 1. The gold random plasmonic nanoparticle arrays were prepared through 1:1 ratio mixing of $0.05 \mathrm{mg}^{-1} \mathrm{ml}^{-1}$ water suspended of $20 \mathrm{~nm}$ gold nano-particles (PlasmaChem $\mathrm{GmbH}$ ) with polyvinyl alcohol (PVA) water solution at a concentration of $34 \mathrm{mg} \cdot \mathrm{ml}^{-1}$ then spin-cast onto a glass substrate to form a film of $30 \mathrm{~nm}$ thickness. The active layer was prepared by doping the molecular laser dye Pyridine 2 at a concentration of $0.5 \mathrm{mg} \cdot \mathrm{ml}^{-1}$ into ethanol solution containing the polymer poly (methacrylic acid) (PMA) at a concentration of $9 \mathrm{mg} \cdot \mathrm{ml}^{-1}$ then spin-cast onto the plasmonic array to form a film of $10 \mathrm{~nm}$ thickness.

Fluorescence spectra were measured using far field spectroscopy. Dark field excitation was provided using $405 \mathrm{~nm}$ with excitation density $200 \mathrm{~W} . \mathrm{cm}^{-2}$. Emission was collected at normal incidence from the sample surface using a 0.26 numerical aperture objective lens and directed toward a $0.25 \mathrm{~m}$ nitrogen-cooled charge-coupled device (CCD).

\section{Results and discussion}

Figure 2 (a) shows an AFM image of a $20 \mathrm{~nm}$ gold nanoparticle plasmonic array embedded in $30 \mathrm{~nm}$ PVA thick layer. As can be seen in Fig. 2(a) and (b) the gold nanoparticles are randomly 
distributed on the PVA surface with tendency to form clusters on a small scale. From Fig. 2(a) it is clear that some of the gold nanoparticles penetrate over the PVA surface. It is important to note that the AFM image only shows the particles distribution at the topmost surface that in a direct contact with the Pyridine 2/PMA layer. From the AFM results in Fig. 2(a) it is clear that the gold nano-particles have a random distribution in the vertical direction of the PVA layer.

Figure 3(a) shows the emission spectra of Pyridine 2 measured from three different locations on the plasmonic array surface. The variation in the integrated emission intensity between these three different locations is less than $10 \%$, illustrating that the gold nanoparticles are evenly distributed within the sample. In Fig. 3(b) we plot the fluorescence emission spectrum of a control film of Pyridine 2 in PMA along with the averaged emission spectrum of Pyridine 2 (red curve) recorded from three different locations on the plasmonic array surface. As can be seen, the fluorescence emission recorded from the plasmonic array is significantly enhanced compared to the emission intensity of the control film as a result of the coupling between the molecular dipoles and the confined optical field in the underlying plasmonic array. By comparing the integrated emission intensity over all wavelengths from the control film and the plasmonic array we deduced three times enhancements in the emission intensity of Pyridine 2 as a result of coupling between the molecular dipoles and plasmonic array. Our achieved enhancements are similar to that reported by Gopinath et al [13] using quasiperiodic nanoparticle array.

To gain further insight into the observed emission intensity enhancement in Fig. 3 and on the effect of the concentration of the gold nanoparticles within the array on these enhancements, we have used three dimensional finite domain (FDTD) calculations to simulate the optical properties of our structure. Here we ran a series of simulations as a function of the gold nanoparticles concentration/fill factor (FF) in the PVA which ranged from 0.025 to 0.55 . In this work we defined the fill factor as $\mathrm{FF}=N A_{s} / A_{P V A}$, where $N$ is the number of the gold nanoparticles used in the calculations, $A_{s}$ is the gold nanoparticle cross-sectional area and $A_{P V A}$ is the surface area of the PVA layer. In the simulations, we also assumed that the gold nanoparticles are randomly distributed within the volume of the PVA layer with random vertical distribution ranging from the bottom of the PVA layer up to $4 \mathrm{~nm}$ below the centre of the active layer.

(C) 2014, Elsevier. Licensed under the Creative Commons Attribution-NonCommercial-NoDerivatives 4.0 International http://creativecommons.org/licenses/by-nc-nd/4.0/ 
Emission intensity $I\left(\lambda_{\mathrm{em}}\right)$ depends on two factors [30]: the first is the excitation intensity $I\left(\lambda_{\mathrm{ex}}\right)$ and the second is the optical density of states for the emission wavelengths $D\left(\lambda_{\mathrm{em}}\right)$. Thus, in general $I\left(\lambda_{e m}\right) \alpha I\left(\lambda_{e x}\right) D\left(\lambda_{e m}\right)$. The observed enhancements in the emission intensity are due to the present of the plasmonic array. The plasmonic array can enhance the excitation field intensity $I\left(\lambda_{\mathrm{ex}}\right)$ at the position of the emitting dipole and/or modify the optical density of states for the emission wavelengths $D\left(\lambda_{\mathrm{em}}\right)$. Here it is important to notice that in this work, we used $\lambda_{\mathrm{ex}}=405 \mathrm{~nm}$ as the excitation wavelength, which is spectrally far from the plasmonic resonance of the gold nanoparticles used in this work $\left(\lambda_{P R_{\text {gold }}} \geq 530 \mathrm{~nm}\right)$. Therefore, we do not expect the observed intensity enhancements to be due to any modification in the excitation field intensity $I\left(\lambda_{\mathrm{ex}}\right)$ at the position of the emitting dipole. To confirm the above argument, we calculated the enhancements in field intensity at the excitation wavelength $\lambda_{\mathrm{ex}}=405 \mathrm{~nm}$. In the field enhancements simulations, the structure was excited by $\mathrm{z}$ polarised plane waves propagating along the $y$-direction. Electric field enhancements were calculated at the excitation wavelength $\lambda_{\mathrm{ex}}=405 \mathrm{~nm}$ in a cross-section $x z$-plane through the structure centre.

In Fig. 4(a) we plot a side view for the enhancements in the electric field intensity normalised by the intensity of the incident electric field at the excitation wavelength $\lambda_{\mathrm{ex}}=405 \mathrm{~nm}$. As can be seen there is very little field intensity enhancement at the excitation wavelength within the Pyridine 2 layer. Therefore we conclude that the observed enhancements in Fig. 3(b) are predominantly due to the modification in the optical density of states $D\left(\lambda_{\mathrm{em}}\right)$ for the emission wavelengths.

To quantify the enhancements in the emission intensity due to the optical density of states for the emission wavelengths $D\left(\lambda_{\mathrm{em}}\right)$, we used FDTD model that based on full numerical solution of Maxwell's equation. The emissive material in our structure (Pyridine 2) was modeled in the form of a large number of dipole sources polarized in the z-direction that are randomly distributed throughout the centre of the active layer. To mimic the emission spectrum of Pyridine 2, each dipole source was modeled in the form of a Gaussian oscillating pulse centred at $645 \mathrm{~nm}$ with a linewidth of $75 \mathrm{~nm}$. At each value of FF we considered three possible random distributions for the gold nanoparticles in the PVA layer and thus each simulated emission spectrum presented in this work is the average result of these three distributions. In the calculations, boundary conditions were implemented by introducing a perfect matching layer around the structure and Lorentz-Drude model was used to describe the optical

(C) 2014, Elsevier. Licensed under the Creative Commons Attribution-NonCommercial-NoDerivatives 4.0 International http://creativecommons.org/licenses/by-nc-nd/4.0/ 
constants of gold. In the calculations we employed a grid spacing of $1 \mathrm{~nm}$ in each direction. Emission intensity spectra $I\left(\lambda_{\mathrm{em}}\right)$ then extracted through spatially integrating the Poynting vector in the normal direction over the top plane of the structure. Enhancements in the emission intensity then calculated $\int_{\lambda_{1}}^{\lambda_{2}} I_{\text {plasmonic }}\left(\lambda_{e m}\right) d \lambda$

using $E E=\frac{\lambda_{1}}{\int_{\lambda_{1}}^{\lambda_{2}} I_{0}\left(\lambda_{e m}\right) d \lambda}$ where $I_{\text {plasmonic }}\left(\lambda_{e m}\right)$ is the emission spectrum with the plasmonic array, $I_{0}\left(\lambda_{e m}\right)$ is the emission spectrum without the plasmonic array, $\lambda_{1}=550 \mathrm{~nm}$ and $\lambda_{2}=850 \mathrm{~nm}$.

In Fig. 4(b) we plot the simulated emission spectra as a function of FF over the range 0.025 to 0.55. As can be seen the emission enhancement is a strong function of FF and reaches a maximum value of 7 times at FF $=0.4$ followed by drop with increasing FF further due to the Ohmic losses by the metal. By comparing our experimental enhancements that observed in Fig. 3(b) and the modelled enhancements in Fig. 4(c) we estimated the FF of our fabricated plasmonic array to be around 0.1.

\section{Conclusion}

In summary, we have developed a solution processed approach to construct plasmonic nanoparticle arrays that are capable of strongly enhancing the emission intensity of a fluorescent molecular dye placed into close proximity to the array. Our finite difference time domain (FDTD) simulations showed that randomly distributed $20 \mathrm{~nm}$ gold nanoparticle arrays in polyvinyl alcohol (PVA) layer have the potential to enhance the emission intensity by more than 7 times with a plasmonic array of fill factor $\mathrm{FF}=0.4$. Experimentally we fabricated gold nanoparticle arrays that enhance the emission intensity of the fluorescent molecular dye Pyridine 2 by three times. By comparing our experimental results to the finite difference time domain (FDTD) simulations we estimated the fill factor FF of the fabricated arrays to be around 0.1. These results have important implications in cost-efficient improvement of photonic emitter systems with the possibility of significantly enhancing their performance over the visible and near-infrared spectral range using gold nanoparticles. Also our developed structures can be used as a plasmonic chip to enhance Raman scattering in chemical and bio-sensing applications.

(C) 2014, Elsevier. Licensed under the Creative Commons Attribution-NonCommercial-NoDerivatives 4.0 International http://creativecommons.org/licenses/by-nc-nd/4.0/ 


\section{Acknowledgments}

AE thanks the University of Hull for the award of a PhD studentship.

\section{References}

[1] W. L. Barnes, A. Dereux, T. W. Ebbesen, Nature 424 (2003) 824.

[2] S. A. Maier, H. A. Atwater, J. Appl. Phys. 98 (2005) 011101.

[3] E. J. R. Vesseur, F. J. G. de Abajo, and A. Polman, Phys. Rev. B 82, 165419 (2010)

[4] W. A. Murray and W. L. Barnes, Adv. Mater. 19(2007) 3771

[5] A. V. Akimov, A. Mukherjee, C. L. Yu, D. E. Chang, A. S. Zibrov, P. R. Hemmer, H. Park, M. D.

Lukin, Nature 450 (2007) 402.

(C) 2014, Elsevier. Licensed under the Creative Commons Attribution-NonCommercial-NoDerivatives 4.0 International http://creativecommons.org/licenses/by-nc-nd/4.0/ 
[6] D. E. Chang, A. S. Sørensen, P. R. Hemmer, M. D. Lukin, Phys. Rev. Lett. 97 (2006) 053002.

[7] S. Kumar, A. Huck, U. L. Andersen, Nano Lett. 13 (2013) 1221.

[8] J. N. Anker, W. P. Hall,O. Lyandres, N. C. Shah, J. Zhao and Ri. P. Van Duyne, Nature Mater. 7 (2008) 442.

[9] B. Yu, S. Goodman, A. Abdelaziz, and D. M. O’Carroll, Appl. Phys. Lett. 101 (2012) 151106.

[10] C. Hägglund, G. Zeltzer, R. Ruiz, I. Thomann, H. B. R. Lee, M. L. Brongersma and S. F. Bent, Nano Lett. 13 (2013) 3352.

[11] Q. Gan , F. J. Bartoli , Z. H. Kafafi, Adv. Mater. 25 (2013) 2385.

[12] K. J. Russell, Tsung-Li Liu, S. Cui, E. L. Hu, Nat. Photonics 6 (2012) 459.

[13] A. Gopinath, S. V. Boriskina, S. Yerci, R. Li, L. Dal Negro, Appl. Phys. Lett. 96 (2010) 071113.

[14] F. Xie, J. S. Pang, A. Centeno, M. P. Ryan, D. Jason Riley, N. M. Alford, Nano Research 6 (2013) 496.

[15] J. Henson, J. DiMaria, E. Dimakis, T. D. Moustakas, R. Paiella, Opt. Let. 37 (2012) 79.

[16] B. Ding, C. Hrelescu, N. Arnold, G. Isic, T. A. Klar, Nano Lett.13 (2013) 378.

[17] J. S. Biteen, N. S. Lewis, H. A. Atwater, Appl. Phys. Lett. 88 (2006) 131109.

[18] K. Y. Yang, K. C. Choi, C. W. Ahn, Appl. Phys. Lett. 94 (2009) 173301.

[19] Y. Zhang, K. Aslan, M. J. R. Previte, Appl. Phys. Lett. 90 (2007) 173116.

[20] K. Matsuda, Y. Ito and Y. Kanemitsu, Appl. Phys. Lett. 92 (2008) 211911.

[21] M. H. Chowdhury, K. Ray, S. K. Gray, J. Pond, J. R. Lakowicz, Anal. Chem. 81(2009) 1397.

[22] K. Ray, M. H. Chowdhury, J. R. Lakowicz, Anal. Chem. 79 (2007) 6480.

[23] I. M. Soganci, S. Nizamoglu, E. Mutlugun, O. Akin, H. V. Demir, Opt. Express 15 (2007) 14289.

[24] S. G. Zhang, X. W. Zhang, Z. G. Yin, J. X. Wang, J. J. Dong, H. L. Gao, F. T. Si, S. S. Sun, Y. Tao, Appl. Phys. Lett. 99 (2011) 181116.

[25] A. Kumar, R. Srivastava, D. S. Mehta, M. N. Kamalasanan, Org. Electronics 13 (2012) 1750.

[26] Feng Liu, Jean-Michel Nunzi, Org. Electronics 13 (2012) 1623.

[27] Y. Xiao, J. P. Yang, P. P. Cheng, J. J. Zhu, Z. Q. Xu, Appl. Phys. Lett. 100 (2012) 013308.

[28]Y. Jin, J. Feng, X-L. Zhang, Y-G. Bi, Y. Bai, L. Chen, T. Lan, Y-F. Liu, Q-D. Chen, H-B. Sun, Adv. Mater. 24 (2012)1187.

[29] The 3D FDTD code (CrystalWave) used in this work is a product of Photon Design Ltd, http://www.photond.com.

(C) 2014, Elsevier. Licensed under the Creative Commons Attribution-NonCommercial-NoDerivatives 4.0 International http://creativecommons.org/licenses/by-nc-nd/4.0/ 
[30] S. V. Gaponenko, Introduction to Nanophotonics, chapter 16, Cambridge university press, Cambridge, 2010.

\section{Figure captions}

Fig. 1 (color on line) Schematic drawing of the plasmonic nanoparticle arrays investigated in this work.

Fig. 2 (color on line) (a) AFM image of plasmonic nanoparticle array (b) High magnification image of a cluster region on the plasmonic array surface.

Fig. 3 (color on line) (a) Fluorescence emission spectra of Pyridine 2 measured from three different locations on the plasmonic array surface. (b) Fluorescence emission of a control film of the molecular dye Pyridine 2 in PMA (black curve) together with the fluorescence emission of the Pyridine 2 when coated onto the plasmonic array surface.

(c) 2014, Elsevier. Licensed under the Creative Commons Attribution-NonCommercial-NoDerivatives 4.0 International http://creativecommons.org/licenses/by-nc-nd/4.0/ 
Fig. 4. (color on line) Simulated spatial distribution of the electric field intensity enhancements at the excitation wavelength $\lambda_{\mathrm{ex}}=405 \mathrm{~nm}$ (a). (b) Simulated flouresence emission spectra of the the molecular dye Pyridine 2 generated using FDTD modelling as a function of the plasmonic array fill factor. Insert shows the calculated total enhancements as a function of the plasmonic array fill factor.

(C) 2014, Elsevier. Licensed under the Creative Commons Attribution-NonCommercial-NoDerivatives 4.0 International http://creativecommons.org/licenses/by-nc-nd/4.0/ 


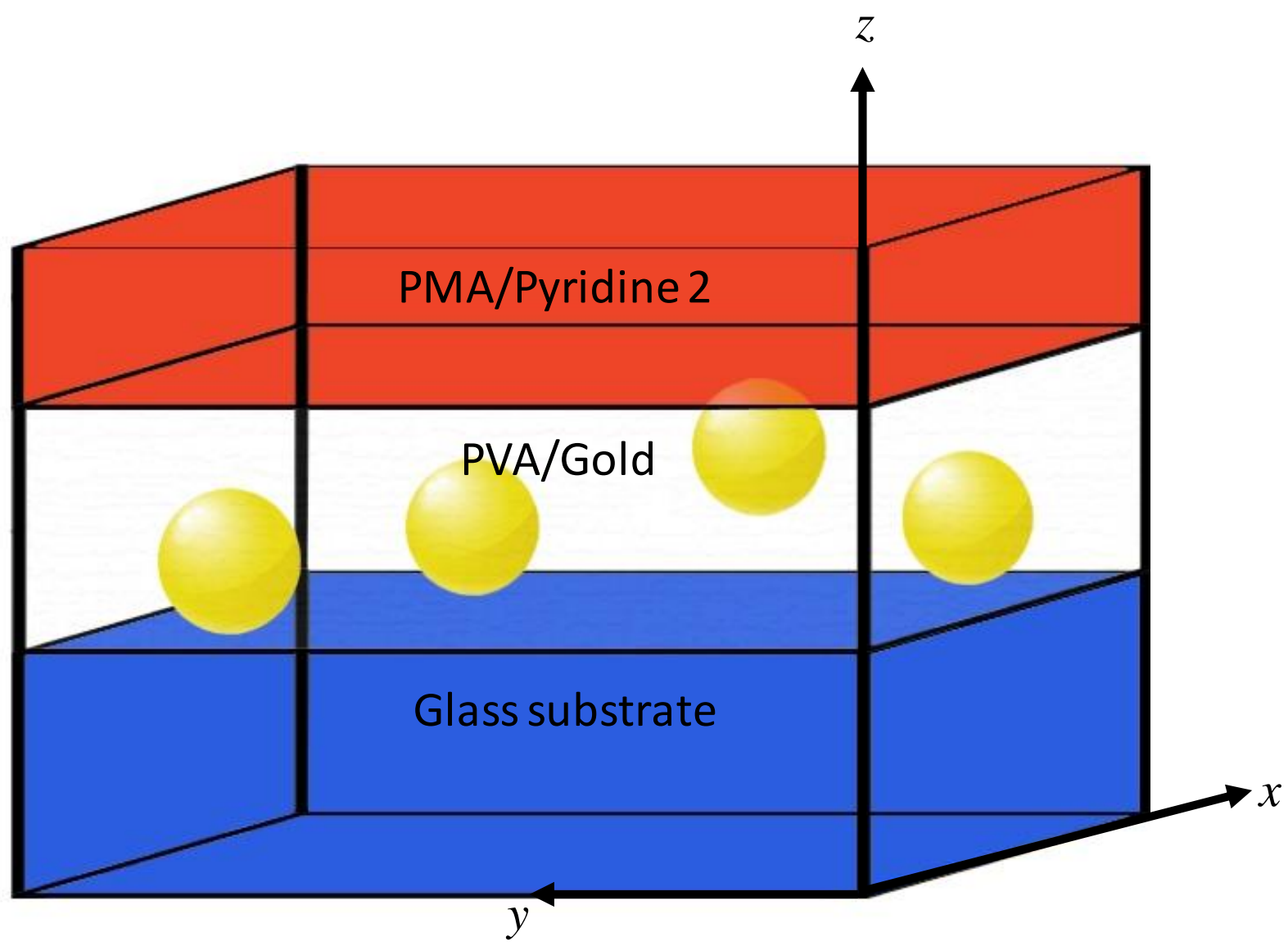

Fig. 1 (color on line) Schematic drawing of the plasmonic nanoparticle arrays investigated in this work.

(C) 2014, Elsevier. Licensed under the Creative Commons Attribution-NonCommercial-NoDerivatives 4.0 International http://creativecommons.org/licenses/by-nc-nd/4.0/ 

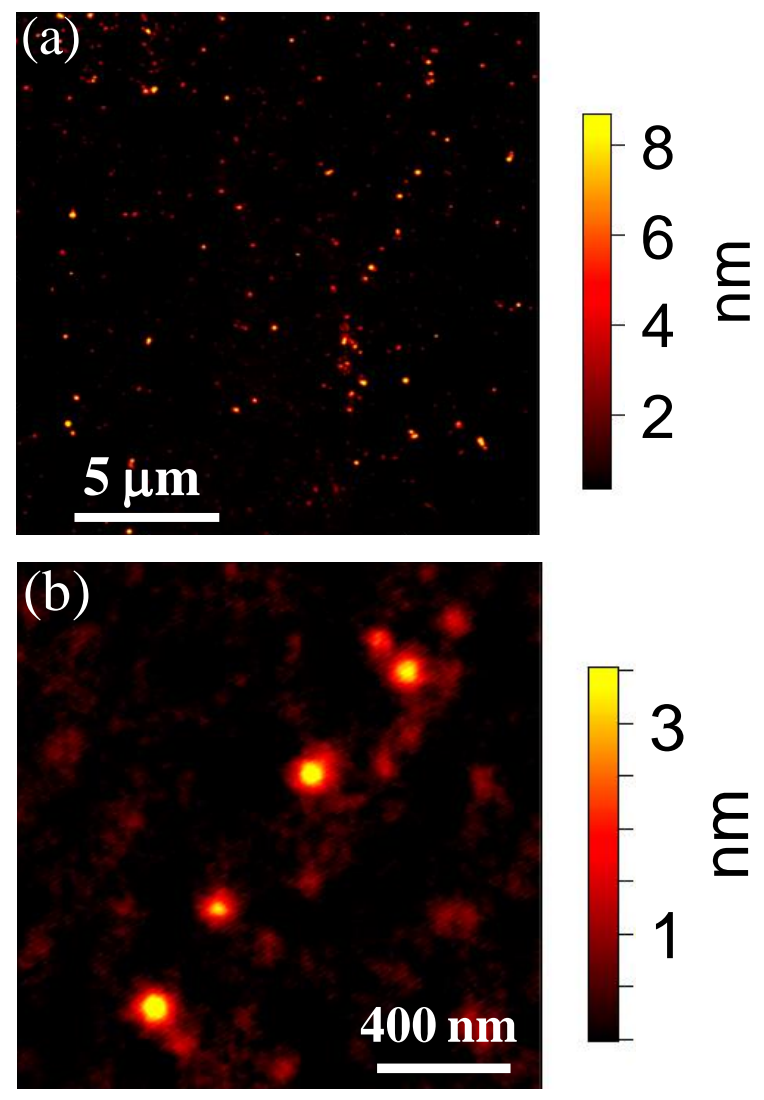

Fig. 2 (color on line) (a) AFM image of plasmonic nanoparticle array (b) High magnification image of a cluster region on the plasmonic array surface.

(C) 2014, Elsevier. Licensed under the Creative Commons Attribution-NonCommercial-NoDerivatives 4.0 International http://creativecommons.org/licenses/by-nc-nd/4.0/ 


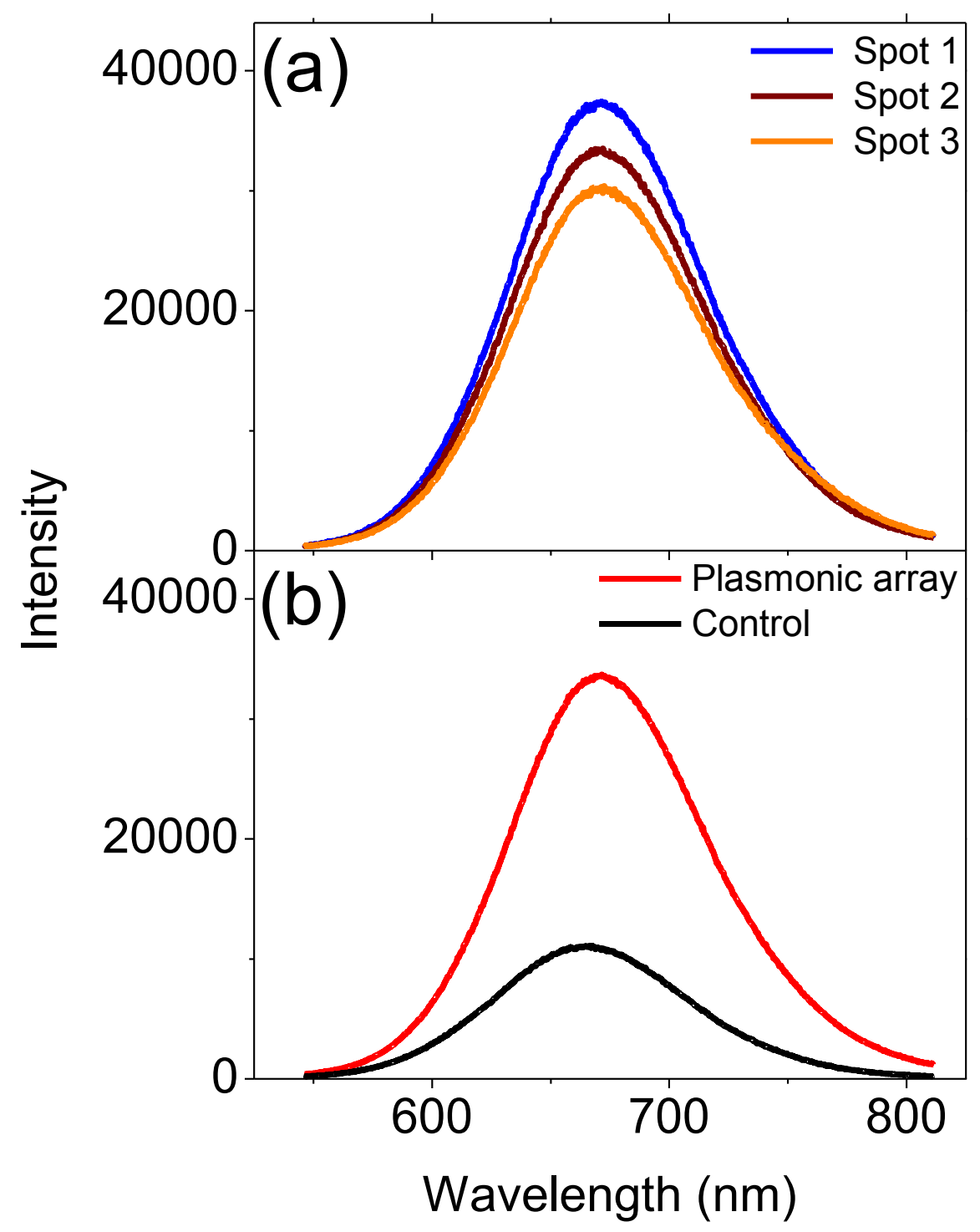

Fig. 3 (color on line) (a) Fluorescence emission spectra of Pyridine 2 measured from three different locations on the plasmonic array surface. (b) Fluorescence emission of a control film of the molecular dye Pyridine 2 in PMA (black curve) together with the fluorescence emission of the Pyridine 2 when coated onto the plasmonic array surface.

(C) 2014, Elsevier. Licensed under the Creative Commons Attribution-NonCommercial-NoDerivatives 4.0 International http://creativecommons.org/licenses/by-nc-nd/4.0/ 

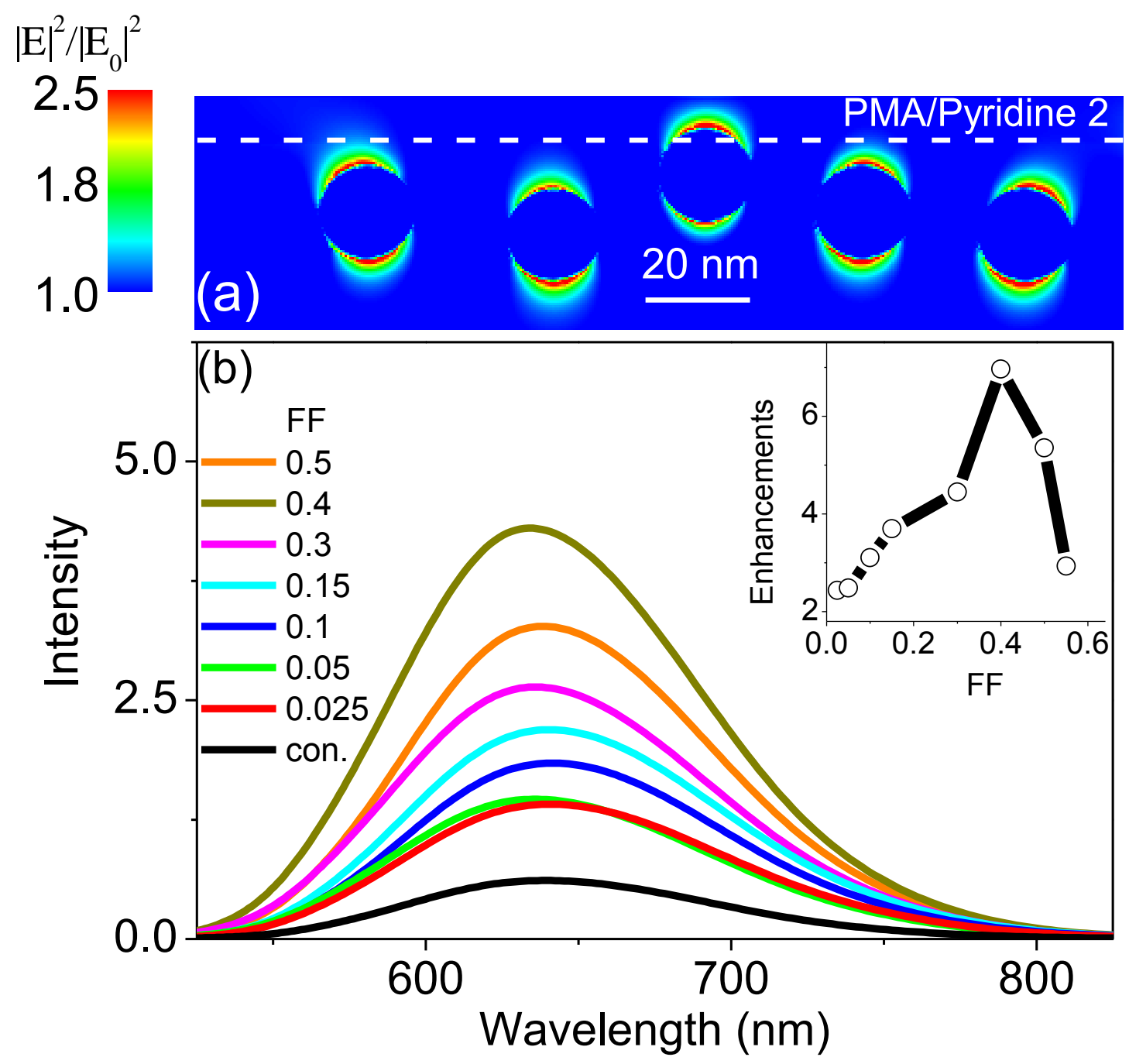

Fig. 4. (color on line) Simulated spatial distribution of the electric field intensity enhancements at the excitation wavelength $\lambda_{\mathrm{ex}}=405 \mathrm{~nm}$ (a). (b) Simulated flouresence emission spectra of the the molecular dye Pyridine 2 generated using FDTD modelling as a function of the plasmonic array fill factor. Insert shows the calculated total enhancements as a function of the plasmonic array fill factor.

(C) 2014, Elsevier. Licensed under the Creative Commons Attribution-NonCommercial-NoDerivatives 4.0 International http://creativecommons.org/licenses/by-nc-nd/4.0/ 